\title{
A Specialized Lightweight Metamorphic Function for KASUMI Metamorphic Cipher and Its FPGA Implementation
}

\author{
Rabie A. Mahmoud \\ Arab Academy for Science, Technology \& Maritime Transport (AASTMT), Latakia Branch, Syria. \\ E-mail: rabiemah@yahoo.com \\ A. Baith Mohamed \\ Arab Academy of Science, Technology \& Maritime Transport (AASTMT), Alexandria, Egypt. \\ E-mail: baithmm@hotmail.com
}

\begin{abstract}
To enhance the performance of the KASUMI Metamorphic Cipher, we apply a lightweight Metamorphic Structure. The proposed structure uses four lightweight bit-balanced operations in the function Meta-FO of the KASUMI Metamorphic Cipher. These operations are: XOR, INV, XNOR, and NOP for bitwise $\mathrm{XOR}$, invert, XNOR, and no operation respectively building blocks of the Specialized Crypto Logic Unit (SCLU). In this work, we present a lightweight KASUMI Specialized-Metamorphic Cipher. In addition, we provide a Field Programmable Gate Array (FPGA) implementation of the proposed algorithm modification.
\end{abstract}

Index Terms-KASUMI, Metamorphic, Lightweight, Cipher, FPGA.

\section{INTRODUCTION}

The KASUMI Metamorphic Cipher [1] is a modified Feistel block cipher from KASUMI cipher [2], [3], [4], [5] which is a 64-bit block cipher using a 128-bit key with eight rounds and nonlinear S-boxes where KASUMI cipher forms the heart of the confidentiality and integrity algorithms of signalling and user data security within the Global Systems for Mobile Communications (GSM), General Packet Radio Service (GPRS), Enhanced Data Rates for GSM Evolution (EDGE), and the Third Generation Mobile System (3GPP) specifications for the Universal Mobile Telecommunications System (UMTS) networks. In this work, we present the KASUMI Specialized-Metamorphic Cipher to encrypt a 64-bit plaintexts using a 128-bit key. It is a lightweight metamorphic cipher that combines the specialized crypto logic unit with the function FO of KASUMI Cipher to encrypt 64-bit plaintext packets using 128-bit key. The Specialized Crypto Logic Unit (SCLU) is a special form of the Generalized Crypto Logic Unit (GCLU) [6] and Crypto Logic Unit (CLU) of the Stone Metamorphic Cipher [7], [8], [9] which are used in many famous ciphers to increase the cipher's entropy and improve its security such as the Metamorphic Twofish Cipher [10], the
Metamorphic MARS Cipher [11], and the Metamorphic-Key-Hopping GOST Cipher [12]. This SCLU is built using four lightweight low-level bit-balanced operations: XORing a key bit with a plaintext bit (XOR), inverting a plaintext bit (INV), XNORing a key bit with a plaintext bit (XNOR), and producing a plaintext bit without any change (NOP). In the following few sections, we provide the structure of the KASUMI Specialized-Metamorphic Cipher by describing the SCLU and the enhanced function Specialized-Meta-FO. Subsequently, we discuss the results of the FPGA implementation of the KASUMI Specialized-Metamorphic Cipher including comparisons among modified KASUMI ciphers, a summary and our conclusions.

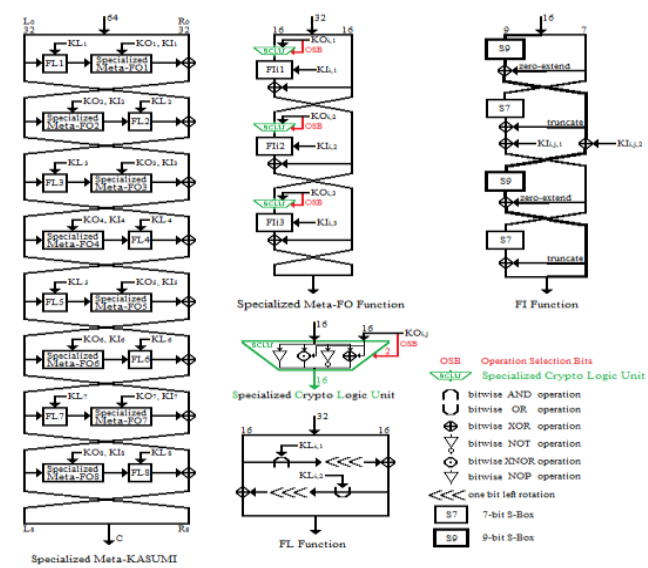

Fig.1. The structure of the KASUMI Specialized-Metamorphic Cipher

\section{THE KASUMI SPECIALIZED-METAMORPHIC STRUCTURE}

The KASUMI Specialized-Metamorphic cipher is a lightweight form of KASUMI Metamorphic cipher, which is a Feistel Cipher with eight rounds encrypting 64-bit plaintext packets using 128-bit key, by replacing the crypto logic unit in the function Meta-FO with specialized 
crypto logic unit converting the function Meta-FO into a Specialized-Meta-FO. Figure 1 shows the block diagram of the proposed KASUMI Specialized-Metamorphic Cipher.

\section{A. $\quad$ The Specialized Crypto Logic Unit (SCLU)}

The Specialized Crypto Logic Unit (SCLU) is a round key-dependent special function modified from key-driven Stone Metamorphic cipher and Generalized Crypto Logic Unit (GCLU) by selecting the four most lightweight operations from the GCLU operations. The four lightweight low-level bit-balanced operations are:

- (XOR) by XORing a key bit with a plaintext bit,

- (INV) by inverting a plaintext bit,

- (XNOR) by XNORing a key bit with a plaintext bit,

- (NOP) by producing the plaintext without any change.

Figure 2 shows the specialized crypto logic unit SCLU and Table 1 demonstrates each one of SCLU operations.

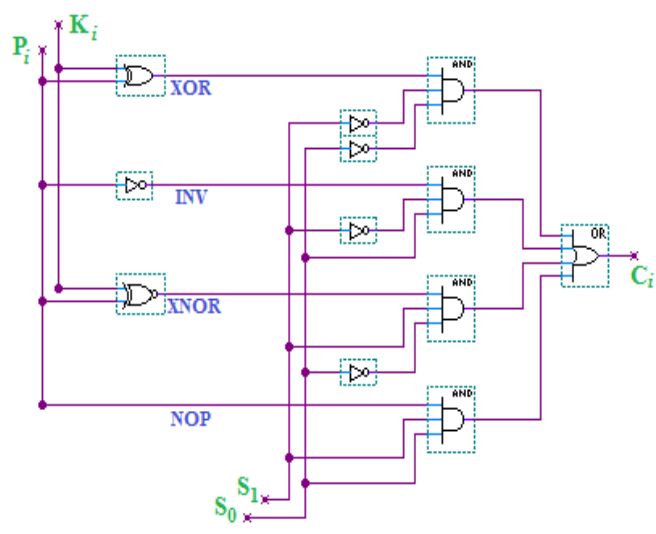

Fig.2. The specialized Crypto Logic Unit (SCLC)

Table 1. SCLU Operations

\begin{tabular}{|c|c|c|}
\hline Mnemonic & Operation & Select Operation code \\
\hline XOR & $C_{i}=K_{i} \oplus P_{i}$ & "00" \\
\hline INV & $C_{i}=\neg\left(P_{i}\right)$ & "01" \\
\hline XNOR & $C_{i}=K_{i} \odot P_{i}$ & "10" \\
\hline NOP & $C_{i}=P_{i}$ & "11" \\
\hline
\end{tabular}

This SCLU is used as the encryptor and the decryptor where by changing the output cipher bit to become an input plain text bit, the new output will be the same as the old plain text bit. Appendix A shows the truth table of SCLU. Likewise, the operation selection bits (S1 S0) can be chosen from any two sub-key bits where the operation selection bits in the KASUMI Specialized-Metamorphic cipher are chosen from the $\mathrm{KO}_{\mathrm{i}, \mathrm{j}}$ round keys. Figure 3 shows the location of operation selection bits.

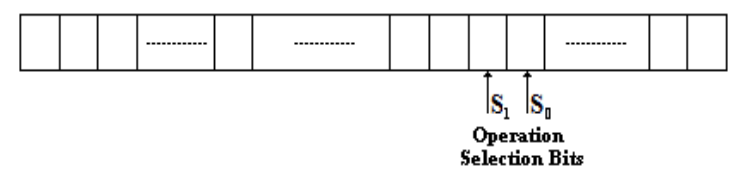

Fig.3. The Proposed Key Format Where The Location Of The Operation Selection Bits Is Shown

\section{B. The Function Specialized-Meta-FO}

The input to the function Specialized-Meta-FO comprises

- 32-bit data input $\boldsymbol{I}$,

- 48-bit subkey $\boldsymbol{K O}_{\boldsymbol{i}}$,

- 48-bit subkey $\boldsymbol{K I}_{\boldsymbol{i}}$.

The 32-bit data input is split into two halves, $\boldsymbol{L}_{\boldsymbol{0}}$ and $\boldsymbol{R}_{\boldsymbol{0}}$ where

$$
I=L_{0} \| R_{0}
$$

The 48-bit subkeys are subdivided into three 16-bit subkeys where

$$
K O_{i}=K O_{i, 1}\left\|K O_{i, 2}\right\| K O_{i, 3} \text { and } K I_{i}=K I_{i, 1}\left\|K I_{i, 2}\right\| K I_{i, 3},
$$

and so for each integer $\boldsymbol{j}$ with $1 \leq \boldsymbol{j} \leq 3$ chose from $\boldsymbol{K} \boldsymbol{O}_{i, j}$ 2-bit operation_selection_bits (OSB).

We define

If operation_selection_bits $=$ “ 00 ” then

$$
\begin{gathered}
\boldsymbol{R}_{j}=\boldsymbol{F I}\left(\boldsymbol{L}_{j-1} \oplus K \boldsymbol{O}_{i, j}, K I_{i, j}\right) \oplus \boldsymbol{R}_{j-1} \\
L_{j}=R_{j-1}
\end{gathered}
$$

If operation_selection_bits $=$ “01" then

$$
\begin{gathered}
\boldsymbol{R}_{j}=\boldsymbol{F I}\left(\neg \boldsymbol{L}_{j-1}, K I_{i, j}\right) \oplus \boldsymbol{R}_{j-1} \\
\boldsymbol{L}_{j}=\boldsymbol{R}_{j-1}
\end{gathered}
$$

If operation_selection_bits $=$ “10” then

$$
\begin{gathered}
R_{j}=F I\left(L_{j-1} \odot K O_{i, j}, K I_{i, j}\right) \oplus R_{j-1} \\
L_{j}=R_{j-1}
\end{gathered}
$$

If operation_selection_bits $=$ " 11 " then

$$
\begin{aligned}
& R_{j}=F I\left(L_{j-1}, K I_{i, j}\right) \oplus R_{j-1} \\
& \boldsymbol{L}_{j}=\boldsymbol{R}_{j-1}
\end{aligned}
$$

Finally, we return the 32-bit value $\left(\boldsymbol{L}_{\mathbf{3}} \| \boldsymbol{R}_{\mathbf{3}}\right)$.

\section{THE FPGA IMPLEMENTATION}

The KASUMI Specialized-Metamorphic Cipher FPGA-based implementation is applied to encrypt 64-bit plaintext packet using 128-bit user key producing 64-bit ciphertext packet at each cycle. We have implemented the cipher applying the VHDL hardware description language 2008 version [13], [14], [15] and utilizing Altera design environment Quartus II 15.0 (64-bit) Web Edition [16] with ModelSim Altera Starter Edition 10.3d [17]. The FPGA design was implemented using EP4CGX50DF27C6, Cyclone IV GX family device. The schematic diagram of proposed cipher with the implementation results is shown in Figure 4. RTL screen of the FPGA implementation is shown in Figure 5. Figure 
6 shows the Technology Map Viewer for part of the hardware implementation of the cipher. Figure 7 demonstrates the floor plan for proposed modified cipher and Figure 8 displays the simulation showing the input, key, and the output cipher text bits. Appendix B displays the analysis and synthesis summary and timing analyzer of KASUMI Specialized-Metamorphic cipher. Major synthesis and timing differences among KASUMI, the KASUMI Metamorphic, the KASUMI Generalized-Metamorphic, and the KASUMI Specialized-Metamorphic ciphers in the balanced optimization technique are shown in Appendix C. Appendix D demonstrates the design and sample VHDL code of the function Specialized-Meta-FO.

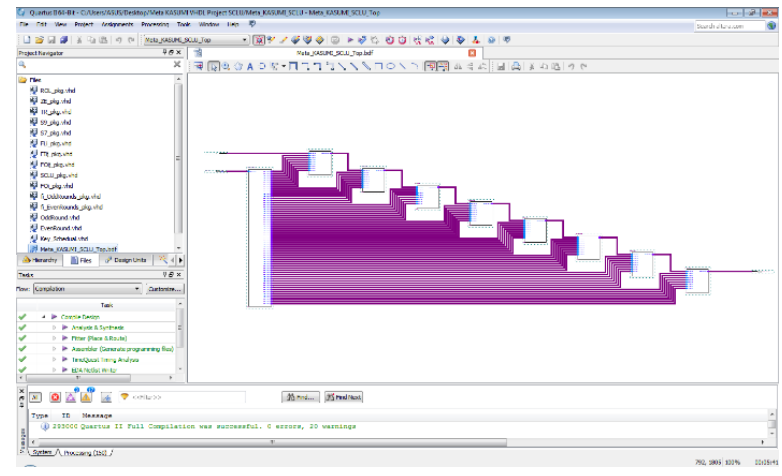

Fig.4. Compiler Tool Screen Showing Correct Implementation and Schematic Diagram of KASUMI Specialized-Metamorphic Cipher

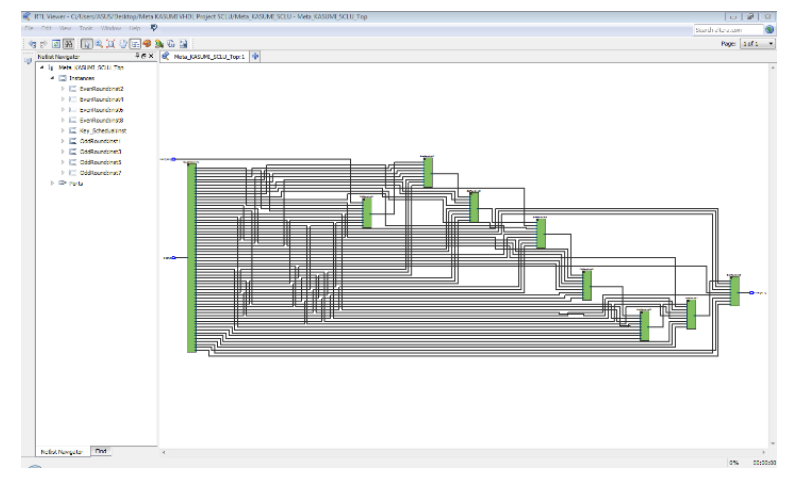

Fig.5. RTL Screen of KASUMI Specialized-Metamorphic Cipher

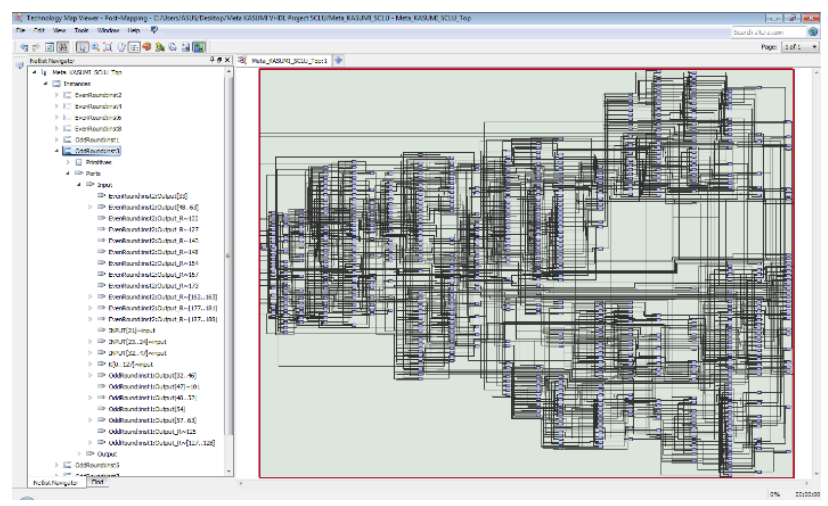

Fig.6. Technology Map Viewer for Part of one Round of KASUMI Specialized-Metamorphic cipher

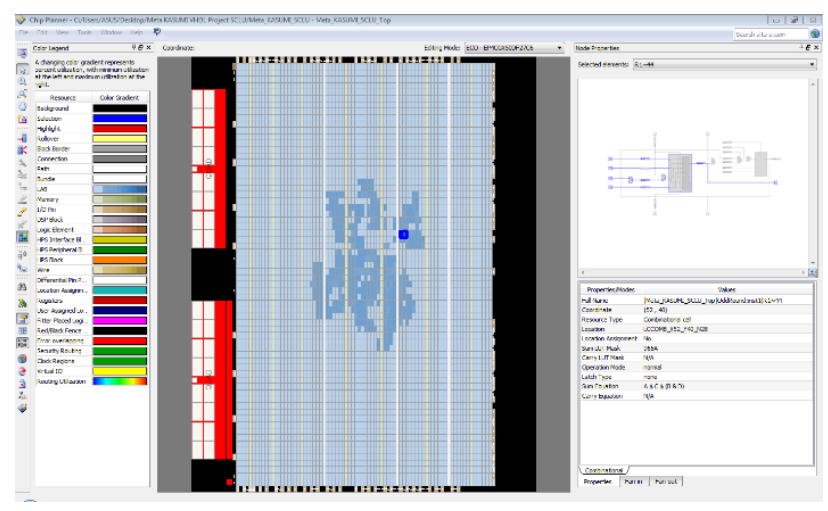

Fig.7. Floor-plan of Chip of KASUMI Specialized-Metamorphic Cipher

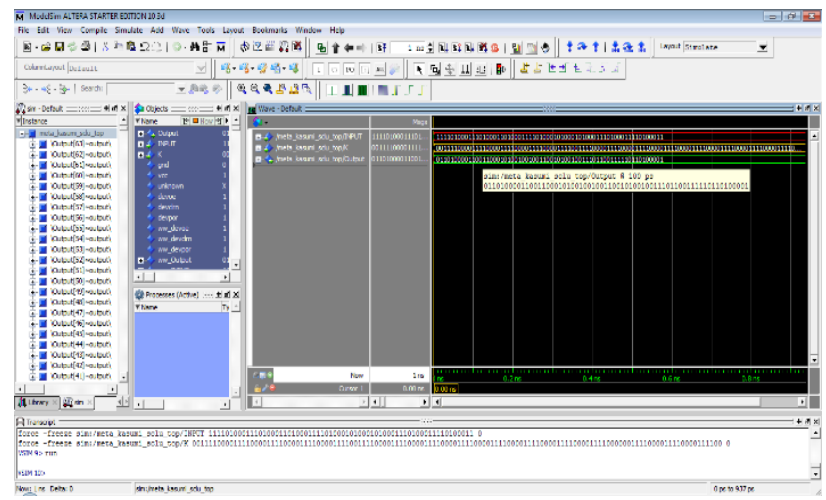

Fig. 8. ModelSim Simulator Screen Showing the Input, Key, and Output of KASUMI Specialized-Metamorphic Cipher

\section{SUMMARY AND CONCLUSIONS}

We have proposed a lightweight modified cipher that is based on the KASUMI Metamorphic cipher. The modified cipher is called the KASUMI Specialized-Metamorphic cipher. A specialized crypto logic unit that utilizing the bit-balanced operations XOR, INV, XNOR, and NOP is merged in each round of the function Meta-FO of KASUMI Metamorphic cipher converting it into lightweight function Specialized-Meta-FO. In addition, we have presented a proof-of-concept FPGA hardware implementation of the proposed cipher. Various FPGA optimization techniques namely Balanced, High Performance Effort, Aggressive Performance, High Power Effort, Aggressive Power, and Aggressive Area optimization techniques were compared for proposed ciphers. Moreover, resources and timing delays comparisons between the KASUMI, the KASUMI Metamorphic, the KASUMI Generalized-Metamorphic, and the KASUMI Specialized-Metamorphic were shown. Certainly, KASUMI Specialized-Metamorphic cipher increased security with less consuming resources than KASUMI cipher itself. 
APPENDIX A THE TRUTH TABLE OF THE SCLU

\begin{tabular}{|c|c|c|c|c|c|}
\hline $\mathrm{P}_{\mathrm{i}}$ & $\mathrm{K}_{\mathrm{i}}$ & $\mathrm{S}_{1}$ & $\mathrm{~S}_{0}$ & Operation & $\mathrm{C}_{\mathrm{i}}$ \\
\hline 0 & 0 & 0 & 0 & XOR & 0 \\
\hline 0 & 0 & 0 & 1 & $\mathrm{INV}$ & 1 \\
\hline 0 & 0 & 1 & 0 & $\mathrm{XNOR}$ & 1 \\
\hline 0 & 0 & 1 & 1 & $\mathrm{NOP}$ & 0 \\
\hline 0 & 1 & 0 & 0 & $\mathrm{XOR}$ & 1 \\
\hline 0 & 1 & 0 & 1 & $\mathrm{INV}$ & 1 \\
\hline 0 & 1 & 1 & 0 & $\mathrm{XNOR}$ & 0 \\
\hline 0 & 1 & 1 & 1 & $\mathrm{NOP}$ & 0 \\
\hline 1 & 0 & 0 & 0 & XOR & 1 \\
\hline 1 & 0 & 0 & 1 & $\mathrm{INV}$ & 0 \\
\hline 1 & 0 & 1 & 0 & XNOR & 0 \\
\hline 1 & 0 & 1 & 1 & $\mathrm{NOP}$ & 1 \\
\hline 1 & 1 & 0 & 0 & XOR & 0 \\
\hline 1 & 1 & 0 & 1 & $\mathrm{INV}$ & 0 \\
\hline 1 & 1 & 1 & 0 & XNOR & 1 \\
\hline 1 & 1 & 1 & 1 & NOP & 1 \\
\hline
\end{tabular}

\section{APPENDIX B THE FITTER AND TIMING REPORT DETAILS OF IMPLEMENTING KASUMI SPECIALIZED-METAMORPHIC CIPHER}

FPGA synthesis of KASUMI Specialized-Metamorphic cipher is implemented with no time restrictions in Balanced, High Performance Effort, Aggressive Performance, High Power Effort, Aggressive Power, and Aggressive Area optimization techniques determining the usage number of logic elements, connections, and time delays. Table B1 shows the number of usage logic elements and their interconnections among optimization techniques of implementing KASUMI Specialized-Metamorphic cipher and Table B2 shows the timing delays among optimization techniques related with Slow $1200 \mathrm{mV} 85^{\circ} \mathrm{C}$ Timing Model, Slow $1200 \mathrm{mV} 0^{\circ} \mathrm{C}$ Timing Model, and Fast $1200 \mathrm{mV} 0^{\circ} \mathrm{C}$ Timing Model. Figures B.1 and B.2 show a comparison chart of timing delays for KASUMI Specialized-Metamorphic cipher in Balanced optimization technique implementation.

\section{Analysis \& Synthesis and Fitter Summary}

- Family: Cyclone IV GX

- Device: EP4CGX50DF27C6

- Nominal Core Voltage: $1.20 \mathrm{~V}$

- Minimum Core Junction Temperature: $0{ }^{\circ} \mathrm{C}$

- Maximum Core Junction Temperature: $85^{\circ} \mathrm{C}$.

- Optimization Technique: Balanced

- Total logic elements: 5,521 out of 49,888 (11\%)

-- Combinational with no register: 5,521

-- Register only: 0

-- Combinational with a register: 0

Logic element usage by number of LUT inputs

-- 4 input functions: 4,230

-- 3 input functions: 690

-- <=2 input functions: 601

-- Register only: 0

Logic elements by mode
-- Normal mode: 5,521

-- Arithmetic mode: 0

- Total LABs: 400 out of 3,118 (13\%)

- I/O pins: 256 out of $343(75 \%)$

-- Clock pins: 2 out of $10(20 \%)$

-- Dedicated input: 0 out of $25(0 \%)$

- Total block memory bits: 0 out of 2,562,048 (0 \%)

- Embedded Multiplier 9-bit elements: 0 out of 280 (0\%)

- Maximum fan-out: 34

- Highest non-global fan-out: 34

- Total fan-out: 20,517

- Average fan-out: 3.40

- Average interconnect usage (total/H/V): $4.0 \%$ / 3.6\% / $4.5 \%$

- Peak interconnect usage (total/H/V): $27.7 \%$ / 24.7\% / $32.0 \%$

- Block interconnects: 7,387 out of 232,464 (3\%)

- C16 interconnects: 670 out of 6,642 (10\%)

- C4 interconnects: 4,410 out of 136,080 (3\%)

- Direct links: 906 out of 232,464 $(<1 \%)$

- GXB block output buffers: 0 out of 2,640 (0\%)

- Global clocks: 0 out of 30 (0\%)

- Interquad Reference Clock Outputs: 0 out of $2(0 \%)$

- Interquad TXRX Clocks: 0 out of $16(0 \%)$

- Interquad TXRX PCSRX outputs: 0 out of $8(0 \%)$

- Interquad TXRX PCSTX outputs: 0 out of $8(0 \%)$

- Local interconnects: 3,254 out of 73,920 (4\%)

- R24 interconnects: 580 out of $6,930(8 \%)$

- R4 interconnects: 4,515 out of 190,740 (2\%)

\section{TimeQuest Timing Analyzer Summary}

- Slow $1200 \mathrm{mV} 85^{\circ} \mathrm{C}$ Model

- Longest propagation delay RR which is measured from rising edge to rising edge was $143.172 \mathrm{~ns}$ from input port "K[114]" to output port "Output[47]". Also, longest delay RF which is measured from rising edge to falling edge was $143.277 \mathrm{~ns}$, longest delay FR which is measured from falling edge to rising edge was $143.701 \mathrm{~ns}$, and longest delay FF which is measured from falling edge to falling edge was $143.806 \mathrm{~ns}$.

- Longest minimum propagation delay was from input port "INPUT[13]" to output port "Output[27]" where RR was $25.782 \mathrm{~ns}$, RF was $25.609 \mathrm{~ns}$, FR was $26.290 \mathrm{~ns}$, and FF was $26.117 \mathrm{~ns}$.

\section{- Slow $1200 \mathrm{mV} 0^{\circ} \mathrm{C}$ Model}

- Longest propagation delay was from input port "K[114]" to output port "Output[47]" where RR was $129.061 \mathrm{~ns}, \mathrm{RF}$ was $129.044 \mathrm{~ns}$, FR was $129.381 \mathrm{~ns}$, and FF was 129.364 ns.

- Longest minimum propagation delay was from input port "INPUT[13]" to output port "Output[27]" where RR was $23.243 \mathrm{~ns}$, RF was $23.219 \mathrm{~ns}$, FR was $23.596 \mathrm{~ns}$, and FF was $23.572 \mathrm{~ns}$.

\section{- Fast $1200 \mathrm{mV} 0^{\circ} \mathrm{C}$ Model}


- Longest propagation delay was from input port "K[114]" to output port "Output[47]" where RR was $83.980 \mathrm{~ns}$, RF was $84.262 \mathrm{~ns}$, FR was $84.751 \mathrm{~ns}$, and FF was $85.033 \mathrm{~ns}$.
- Longest minimum propagation delay was from input port "INPUT[13]" to output port "Output[27]" where RR was $14.732 \mathrm{~ns}$, RF was $14.398 \mathrm{~ns}$, FR was $15.395 \mathrm{~ns}$, and FF was $15.061 \mathrm{~ns}$.

Table B1. A resource and Routing Usage Comparison Among Optimization Technique Implementations of KASUMI Specialized-Metamorphic Cipher

\begin{tabular}{|c|c|c|c|c|c|c|c|}
\hline & & Balanced & $\begin{array}{c}\text { High Performance } \\
\text { Effort }\end{array}$ & $\begin{array}{c}\text { Aggressive } \\
\text { Performance }\end{array}$ & $\begin{array}{c}\text { High } \\
\text { Power Effort }\end{array}$ & $\begin{array}{l}\text { Aggressive } \\
\text { Power }\end{array}$ & $\begin{array}{c}\text { Aggressive } \\
\text { Area }\end{array}$ \\
\hline \multicolumn{2}{|c|}{ Total LEs } & 5521 & 5521 & 5525 & 5521 & 5521 & 5521 \\
\hline \multirow{3}{*}{ Functions } & 4 input & 4230 & 4230 & 4222 & 4230 & 4230 & 4230 \\
\hline & 3 input & 690 & 690 & 695 & 690 & 690 & 690 \\
\hline & $<=2$ input & 601 & 601 & 608 & 601 & 601 & 601 \\
\hline \multirow{3}{*}{ Fan-Out } & Total & 20517 & 20517 & 20514 & 20517 & 50517 & 20517 \\
\hline & Max & 34 & 34 & 34 & 34 & 34 & 34 \\
\hline & Average & 3.40 & 3.40 & 3.39 & 3.40 & 3.40 & 3.40 \\
\hline \multirow{6}{*}{ Interconnects } & Block & 7387 & 7306 & 7239 & 7362 & 7209 & 7387 \\
\hline & $\mathrm{C} 16$ & 670 & 628 & 653 & 656 & 588 & 670 \\
\hline & $\mathrm{C} 4$ & 4410 & 3216 & 3210 & 3343 & 3356 & 4410 \\
\hline & Local & 3254 & 3196 & 3201 & 3204 & 3242 & 3254 \\
\hline & $\mathrm{R} 24$ & 580 & 529 & 567 & 543 & 439 & 580 \\
\hline & $\mathrm{R} 4$ & 4515 & 3060 & 3080 & 3366 & 3517 & 4515 \\
\hline \multicolumn{2}{|c|}{ Direct Links } & 906 & 1488 & 1459 & 1406 & 1105 & 906 \\
\hline
\end{tabular}

Table B2. A Timing Delays Comparison Among Optimization Technique Implementations of KASUMI Specialized-Metamorphic Cipher

\begin{tabular}{|c|c|c|c|c|c|c|c|c|}
\hline & & & Balanced & $\begin{array}{c}\text { High Performance } \\
\text { Effort }\end{array}$ & $\begin{array}{c}\text { Aggressive } \\
\text { Performance }\end{array}$ & $\begin{array}{c}\text { High } \\
\text { Power Effort }\end{array}$ & $\begin{array}{l}\text { Aggressive } \\
\text { Power }\end{array}$ & $\begin{array}{l}\text { Aggressive } \\
\text { Area }\end{array}$ \\
\hline \multirow{8}{*}{$\begin{array}{c}\text { Slow } \\
1200 \mathrm{~m} \\
\text { V } 85^{\circ} \mathrm{C} \\
\text { Model }\end{array}$} & \multirow{4}{*}{ Longest } & $\mathrm{RR}$ & 143.172 & 131.178 & 128.260 & 134.018 & 134.307 & 143.172 \\
\hline & & $\mathrm{RF}$ & 143.277 & 131.050 & 128.186 & 133.957 & 134.273 & 143.277 \\
\hline & & FR & 143.701 & 131.857 & 128.899 & 134.515 & 134.861 & 143.701 \\
\hline & & FF & 143.806 & 131.729 & 128.825 & 134.454 & 134.827 & 143.806 \\
\hline & \multirow{4}{*}{$\begin{array}{c}\text { Longest } \\
\text { Min }\end{array}$} & RR & 25.782 & 23.112 & 23.607 & 23.474 & 23.728 & 25.782 \\
\hline & & $\mathrm{RF}$ & 25.609 & 22.946 & 23.701 & 23.489 & 23.649 & 25.609 \\
\hline & & FR & 26.290 & 23.554 & 24.096 & 23.937 & 24.254 & 26.290 \\
\hline & & FF & 26.117 & 23.388 & 24.190 & 23.952 & 24.175 & 26.117 \\
\hline \multirow{8}{*}{$\begin{array}{c}\text { Slow } \\
1200 \mathrm{~m} \\
\mathrm{~V} 0^{\circ} \mathrm{C} \\
\text { Model }\end{array}$} & \multirow{4}{*}{ Longest } & $\mathrm{RR}$ & 129.061 & 117.923 & 115.660 & 120.528 & 120.957 & 129.061 \\
\hline & & $\mathrm{RF}$ & 129.044 & 117.936 & 115.615 & 120.573 & 120.946 & 129.044 \\
\hline & & FR & 129.381 & 118.477 & 116.098 & 120.813 & 121.379 & 129.381 \\
\hline & & $\mathrm{FF}$ & 129.364 & 118.490 & 116.053 & 120.858 & 121.368 & 129.364 \\
\hline & \multirow{4}{*}{$\begin{array}{c}\text { Longest } \\
\text { Min }\end{array}$} & $\mathrm{RR}$ & 23.243 & 20.696 & 21.263 & 21.180 & 21.348 & 23.243 \\
\hline & & RF & 23.219 & 20.646 & 21.219 & 21.127 & 21.390 & 23.219 \\
\hline & & FR & 23.596 & 21.091 & 21.626 & 21.545 & 21.758 & 23.596 \\
\hline & & $\mathrm{FF}$ & 23.572 & 21.041 & 21.582 & 21.492 & 21.800 & 23.572 \\
\hline \multirow{8}{*}{\begin{tabular}{c|} 
Fast \\
$1200 \mathrm{~m}$ \\
$\mathrm{~V} 0^{\circ} \mathrm{C}$ \\
Model
\end{tabular}} & \multirow{4}{*}{ Longest } & RR & 83.980 & 76.456 & 74.539 & 77.714 & 78.158 & 83.980 \\
\hline & & $\mathrm{RF}$ & 84.262 & 76.237 & 74.357 & 77.474 & 78.041 & 84.262 \\
\hline & & FR & 84.751 & 77.236 & 75.373 & 78.507 & 78.886 & 84.751 \\
\hline & & $\mathrm{FF}$ & 85.033 & 77.017 & 75.191 & 78.267 & 78.769 & 85.033 \\
\hline & \multirow{4}{*}{$\begin{array}{c}\text { Longest } \\
\text { Min }\end{array}$} & $\mathrm{RR}$ & 14.732 & 13.269 & 13.408 & 13.143 & 13.625 & 14.732 \\
\hline & & $\mathrm{RF}$ & 14.398 & 13.035 & 13.575 & 13.351 & 13.370 & 14.398 \\
\hline & & FR & 15.395 & 13.864 & 14.055 & 13.760 & 14.314 & 15.395 \\
\hline & & FF & 15.061 & 13.630 & 14.222 & 13.968 & 14.059 & 15.061 \\
\hline
\end{tabular}




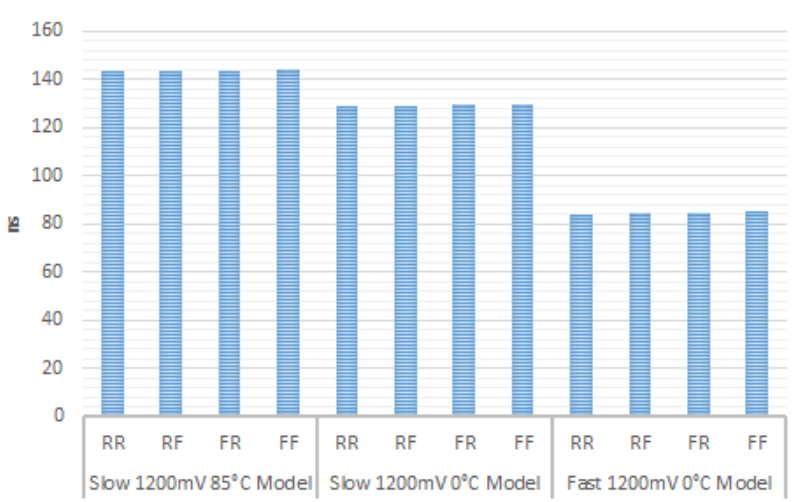

Fig.B.1. Longest Delays of Balanced Optimization Technique Implementation of KASUMI Specialized-Metamorphic Cipher

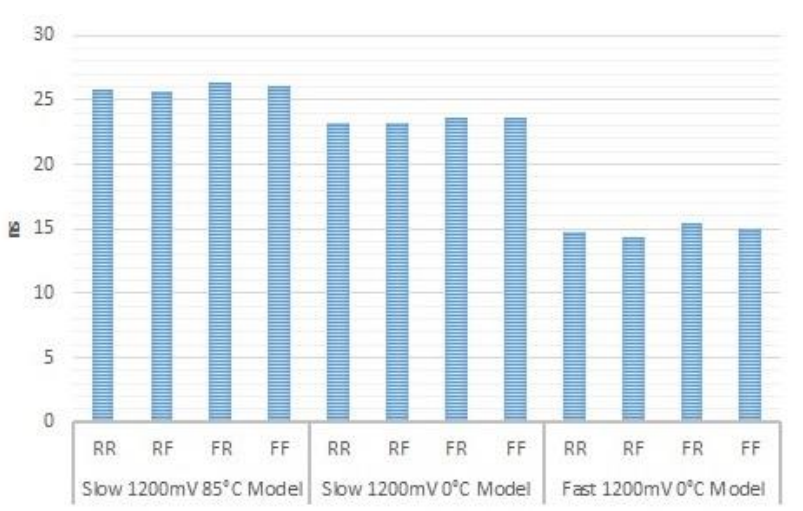

Fig.B.2. Longest Min Delays of Balanced Optimization Technique Implementation of KASUMI Specialized-Metamorphic Cipher
VI. APPENDIX C MAJOR IMPLEMENTING DIFFERENCES AMONG KASUMI SPECIALIZED-METAMORPHIC CIPHER AND MODIFIED KASUMI CIPHERS IN BALANCED OPTIMIZATION TECHNIQUE

KASUMI Specialized-Metamorphic cipher consumes less resources and routing even than KASUMI cipher where the four operations XOR, INV, NOP, and XNOR of specialized crypto logic unit are low synthesized operations and the rising of maximum fan-out implements the KASUMI Specialized-Metamorphic cipher to consume less number of logic elements. Table $\mathrm{C} 1$ shows the number of usage logic elements and their interconnects and Table $\mathrm{C} 2$ shows the timing delays among KASUMI, the KASUMI Metamorphic, the KASUMI Generalized-Metamorphic, and the KASUMI Specialized-Metamorphic ciphers in Balanced optimization technique. Figures C.1 and C.2 show a comparison chart of those timing delays.

Table C1. A resource and Routing Usage Comparison Among KASUMI Specialized-Metamorphic and Modified KASUMI Ciphers

\begin{tabular}{|c|c|c|c|c|c|}
\hline & & KASUMI & Metamorphic-KASUMI & $\begin{array}{c}\text { Generalized-Metamorphic } \\
\text {-KASUMI }\end{array}$ & $\begin{array}{c}\text { Specialized-Metamorphic- } \\
\text { KASUMI }\end{array}$ \\
\hline \multicolumn{2}{|c|}{ Total LEs } & 5582 & 7622 & 10559 & 5521 \\
\hline \multirow{3}{*}{ Functions } & 4 input & 4365 & 5732 & 7759 & 4230 \\
\hline & 3 input & 665 & 1333 & 2026 & 690 \\
\hline & $<=2$ input & 552 & 557 & 774 & 601 \\
\hline \multirow{3}{*}{ Fan-Out } & Total & 20884 & 28366 & 38987 & 20517 \\
\hline & $\operatorname{Max}$ & 31 & 93 & 97 & 34 \\
\hline & Average & 3.42 & 3.48 & 3.52 & 3.40 \\
\hline \multirow{6}{*}{ Interconnects } & Block & 7733 & 10033 & 13633 & 7387 \\
\hline & $\mathrm{C} 16$ & 702 & 783 & 894 & 670 \\
\hline & $\mathrm{C} 4$ & 4773 & 5906 & 8324 & 4410 \\
\hline & Local & 3124 & 4412 & 6142 & 3254 \\
\hline & R24 & 599 & 703 & 846 & 580 \\
\hline & $\mathrm{R} 4$ & 5103 & 5818 & 7819 & 4515 \\
\hline \multicolumn{2}{|c|}{ Direct Links } & 939 & 1193 & 1564 & 906 \\
\hline
\end{tabular}


Table C2. A timing Delays Comparison Among KASUMI Specialized-Metamorphic and Modified KASUMI Ciphers

\begin{tabular}{|c|c|c|c|c|c|c|}
\hline & & & KASUMI & Metamorphic-KASUMI & $\begin{array}{c}\text { Generalized-Metamorphic- } \\
\text { KASUMI }\end{array}$ & $\begin{array}{c}\text { Specialized-Metamorphic-K } \\
\text { ASUMI }\end{array}$ \\
\hline \multirow{8}{*}{$\begin{array}{c}\text { Slow } \\
1200 \mathrm{~m} \\
\text { V } 85^{\circ} \mathrm{C} \\
\text { Model }\end{array}$} & \multirow{4}{*}{ Longest } & RR & 148.443 & 194.146 & 230.329 & 143.172 \\
\hline & & RF & 148.506 & 194.096 & 230.327 & 143.277 \\
\hline & & FR & 149.028 & 194.815 & 231.083 & 143.701 \\
\hline & & FF & 149.091 & 194.765 & 231.081 & 143.806 \\
\hline & \multirow{4}{*}{$\begin{array}{c}\text { Longest } \\
\text { Min }\end{array}$} & RR & 25.630 & 26.335 & 28.950 & 25.782 \\
\hline & & $\mathrm{RF}$ & 25.627 & 26.322 & 28.829 & 25.609 \\
\hline & & FR & 26.143 & 26.891 & 29.433 & 26.290 \\
\hline & & $\mathrm{FF}$ & 26.140 & 26.878 & 29.312 & 26.117 \\
\hline \multirow{8}{*}{$\begin{array}{c}\text { Slow } \\
1200 \mathrm{~m} \\
\mathrm{~V} 0^{\circ} \mathrm{C} \\
\text { Model }\end{array}$} & \multirow{4}{*}{ Longest } & RR & 134.001 & 174.609 & 207.348 & 129.061 \\
\hline & & $\mathrm{RF}$ & 134.175 & 174.587 & 207.314 & 129.044 \\
\hline & & FR & 134.426 & 175.064 & 207.863 & 129.381 \\
\hline & & FF & 134.600 & 175.042 & 207.829 & 129.364 \\
\hline & \multirow{4}{*}{$\begin{array}{c}\text { Longest } \\
\text { Min }\end{array}$} & $\mathrm{RR}$ & 23.116 & 23.697 & 26.042 & 23.243 \\
\hline & & $\mathrm{RF}$ & 23.051 & 23.723 & 26.035 & 23.219 \\
\hline & & FR & 23.483 & 24.152 & 26.410 & 23.596 \\
\hline & & $\mathrm{FF}$ & 23.418 & 24.178 & 26.403 & 23.572 \\
\hline \multirow{8}{*}{$\begin{array}{c}\text { Fast } \\
1200 \mathrm{~m} \\
\mathrm{~V} 0^{\circ} \mathrm{C} \\
\text { Model }\end{array}$} & \multirow{4}{*}{ Longest } & $\mathrm{RR}$ & 87.418 & 113.251 & 135.252 & 83.980 \\
\hline & & RF & 87.090 & 112.990 & 135.013 & 84.262 \\
\hline & & FR & 88.242 & 114.087 & 136.112 & 84.751 \\
\hline & & FF & 87.914 & 113.826 & 135.873 & 85.033 \\
\hline & \multirow{4}{*}{\begin{tabular}{|c|} 
Longest \\
Min
\end{tabular}} & RR & 14.740 & 15.098 & 16.722 & 14.732 \\
\hline & & $\mathrm{RF}$ & 14.455 & 14.835 & 16.399 & 14.398 \\
\hline & & FR & 15.407 & 15.816 & 17.361 & 15.395 \\
\hline & & FF & 15.122 & 15.553 & 17.038 & 15.061 \\
\hline
\end{tabular}

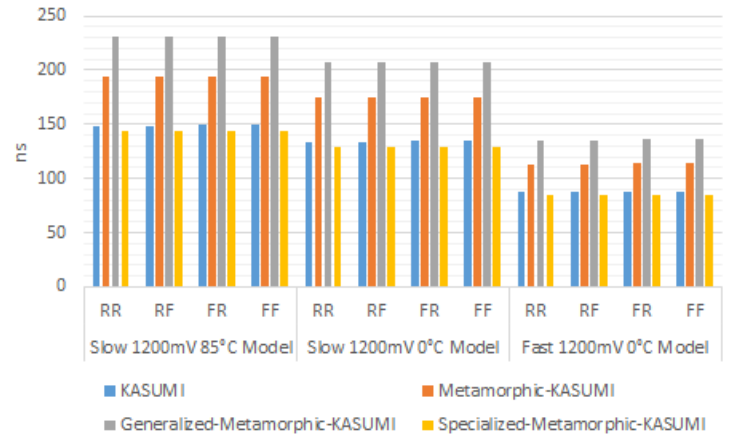

Fig.C.1. Longest Delays of Implementations of Modified KASUMI Ciphers

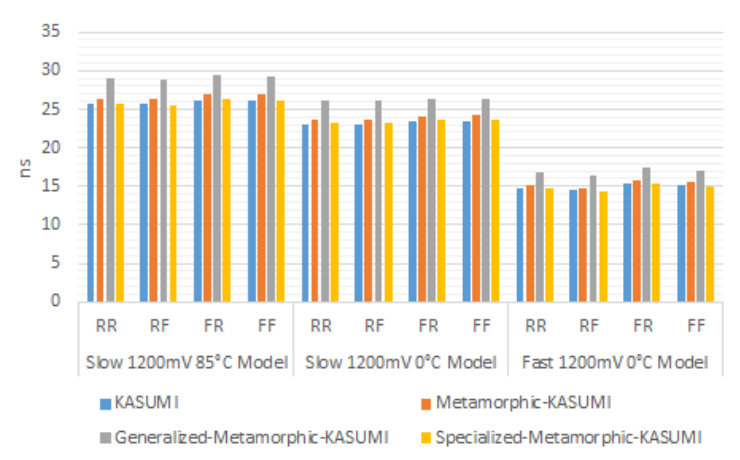

Fig.C.2. Longest Min Delays of Implementations of Modified KASUMI Ciphers

\section{APPENDIX D SAMPLE VHDL CODE OF THE FUNCTION SPECIALIZED-META-FO}

Multi-nested VHDL functions in packages and component configuration of generated statements are used to program Key_Schedual, OddRound, and EvenRound components which are connected together in block diagram/schematic file to implement KASUMI Specialized-Metamorphic cipher. The function Specialized_Meta_FO of KASUMI Specialized-Metamorphic cipher defined through Specialized_Meta_FOi function in Specialized_Meta_FOi_pkg package using SCLU function in SCLU_pkg package and Specialized_Meta_FOij function in Specialized_Meta_FOij_pkg package:

- SCLU function represents the Specialized Crypto Logic Unit with two input parameters 16-bit input and 16-bit KOij. SCLU function returns a 16-bit output after applying the SCLU operations which are related to OSB.

- Specialized_Meta_FOij function represents one round of the three rounds of Specialized_Meta_FOi function where three input parameters 32-bit input, 16-bit KOij, and 16-bit KIij returns a 32-bit output after applying the related operations and calling the FIij and SCLU functions from Flij_pkg, and SCLU_pkg packages respectively. 
- Specialized_Meta_FOi function calls Specialized_Meta_FOij function three times sequentially to implement three rounds and returning a 32-bit output by using seven input parameters 32-bit input, 16-bit KOi1, 16-bit KIi1, 16-bit KIi2, 16-bit KOi2, 16-bit KOi3, and 16-bit KIi3.

Sample VHDL codes are:

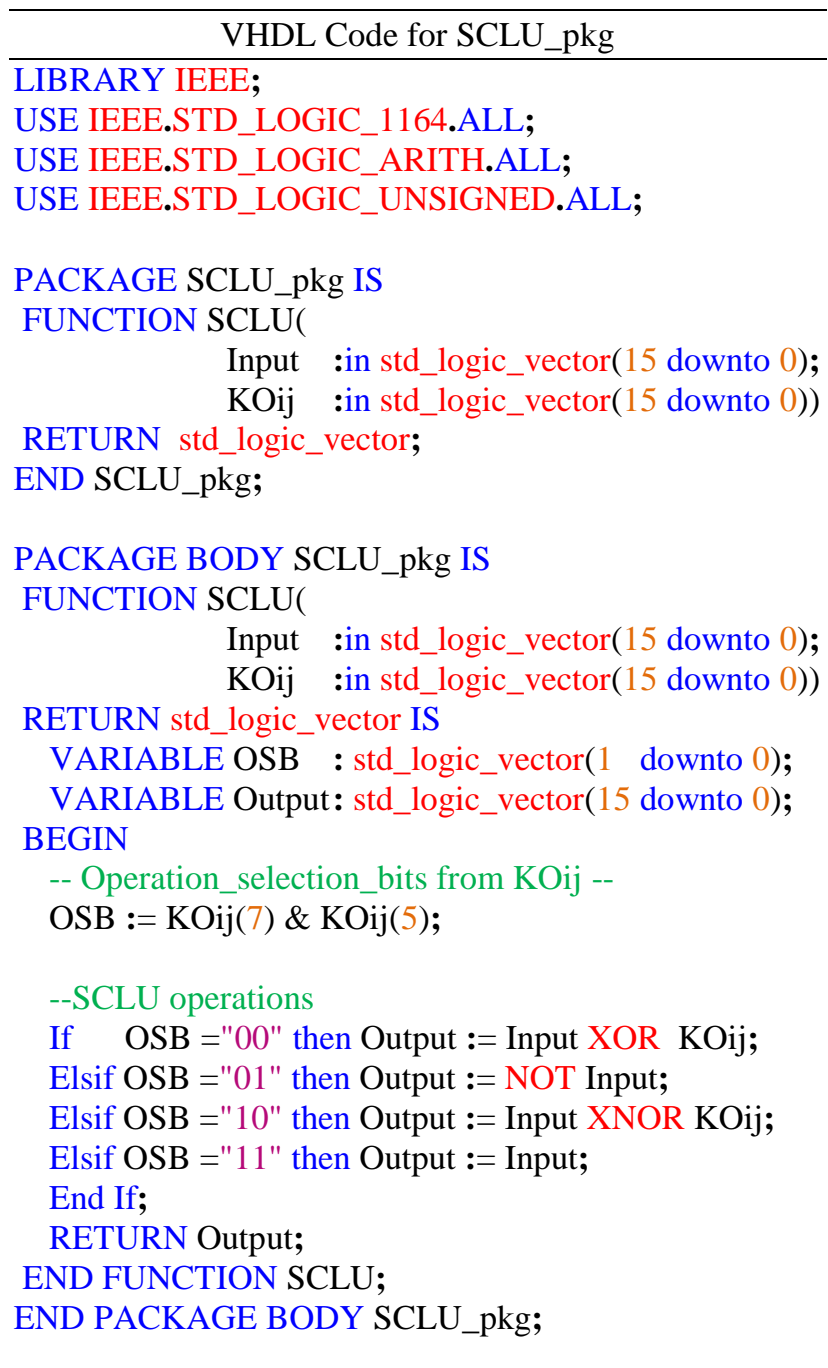

\begin{tabular}{l}
\hline \multicolumn{1}{c}{ VHDL Code for Specialized_Meta_FOij_pkg } \\
\hline LIBRARY IEEE; \\
USE IEEE.STD_LOGIC_1164.ALL; \\
USE IEEE.STD_LOGIC_ARITH.ALL; \\
USE IEEE.STD_LOGIC_UNSIGNED.ALL; \\
-- To call FIij Function -- \\
USE WORK.Flij_pkg.ALL; \\
-- To call SCLU Function -- \\
USE WORK.SCLU_pkg.ALL; \\
PACKAGE Specialized_Meta_FOij_pkg IS \\
FUNCTION Specialized_Meta_FOij ( \\
Input :in std_logic_vector(31 downto 0); \\
KOij :in std_logic_vector(15 downto 0); \\
KIij :in std_logic_vector(15 downto 0))
\end{tabular}

RETURN std_logic_vector;

END Specialized_Meta_FOij_pkg;
PACKAGE BODY Specialized_Meta_FOij_pkg IS

FUNCTION Specialized_Meta_FOij (

Input :in std_logic_vector(31 downto 0); KOij :in std_logic_vector(15 downto 0); KIij :in std_logic_vector(15 downto 0))

RETURN std_logic_vector IS

-- The input halves Lj-1 and Rj-1 --

VARIABLE Input_L : std_logic_vector(15 downto 0);

VARIABLE Input_R : std_logic_vector(15 downto 0);

-- The output halves $\mathrm{Lj}$ and $\mathrm{Rj}$--

VARIABLE Output_L:std_logic_vector(15 downto 0);

VARIABLE Output_R:std_logic_vector(15 downto0);

-- The output of SCLU Function --

VARIABLE Output_SCLU :

std_logic_vector(15 downto 0$)$;

VARIABLE Output : std_logic_vector(31 downto 0); BEGIN

-- Splitting the Input

Input_L := Input(31 downto 16);

Input_R :=Input(15 downto 0$)$;

-- Operation Series

Output_L :=Input_R;

Output_SCLU := SCLU(Input_L, KOij);

Output_R := FIij(Output_SCLU , KIij) XOR Input_R;

-- Output of Function

Output := Output_L \& Output_R;

RETURN Output;

END FUNCTION Specialized_Meta_FOij;

END PACKAGE BODY Specialized_Meta_FOij_pkg;

\begin{tabular}{|c|c|}
\hline \multicolumn{2}{|c|}{ VHDL Code for Specialized_Meta_FOi_pkg } \\
\hline \multicolumn{2}{|l|}{ LIBRARY IEEE; } \\
\hline \multicolumn{2}{|c|}{ USE IEEE.STD_LOGIC_1164.ALL; } \\
\hline \multicolumn{2}{|c|}{ USE IEEE.STD_LOGIC_ARITH.ALL; } \\
\hline \multicolumn{2}{|c|}{ USE IEEE.STD_LOGIC_UNSIGNED.ALL; } \\
\hline \multicolumn{2}{|c|}{-- To call Specialized_Meta_FOij Function -- } \\
\hline \multicolumn{2}{|c|}{ USE WORK. Specialized_Meta_FOij_pkg.ALL; } \\
\hline \multicolumn{2}{|c|}{ PACKAGE Specialized_Meta_FOi_pkg IS } \\
\hline \multicolumn{2}{|c|}{ FUNCTION Specialized_Meta_FOi ( } \\
\hline Input & :in std_logic_vector(31 downto 0); \\
\hline KOi1 & :in std_logic_vector(15 downto 0$)$; \\
\hline KOi2 & :in std_logic_vector(15 downto 0); \\
\hline KOi3 & :in std_logic_vector(15 downto 0$)$; \\
\hline KIi1 & :in std_logic_vector( 15 downto 0$)$; \\
\hline KIi2 & :in std_logic_vector(15 downto 0$)$; \\
\hline KIi3 & :in std_logic_vector(15 downto 0$))$ \\
\hline
\end{tabular}

RETURN std_logic_vector;

END Specialized_Meta_FOi_pkg;

PACKAGE BODY Specialized_Meta_FOi_pkg IS

FUNCTION Specialized_Meta_FOi (

Input :in std_logic_vector(31 downto 0);

KOil :in std_logic_vector(15 downto 0);

KOi2 :in std_logic_vector(15 downto 0);

KOi3 :in std_logic_vector(15 downto 0);

KIi1 :in std_logic_vector(15 downto 0);

KIi2 :in std_logic_vector(15 downto 0);

KIi3 :in std_logic_vector(15 downto 0))

RETURN std_logic_vector IS 
VARIABLE Round1 : std_logic_vector(31 downto 0); VARIABLE Round 2 : std_logic_vector(31 downto 0); VARIABLE Round 3 : std_logic_vector(31 downto 0); VARIABLE Output : std_logic_vector(31 downto 0);

\section{BEGIN}

-- Round 1 of Specialized Meta FOi

Round1 :=Specialized_Meta_FOij(Input, KOi1, KIi1);

-- Round 2 of Specialized_Meta_FOi

Round2 :=Specialized_Meta_FOij(Round1, KOi2, KIi2);

-- Round 3 of Specialized_Meta_FOi

Round3 :=Specialized_Meta_FOij(Round2, KOi3, KIi3);

Output := Round3; -- The Output of Function

RETURN Output;

END FUNCTION Specialized_Meta_FOi;

END PACKAGE BODY Specialized_Meta_FOi_pkg;

\section{REFERENCES}

[1] Rabie A. Mahmoud, A. Baith Mohamed, Magdy Saeb, "Enhancing KASUMI Security by Affixing A Metamorphic Function and The Ensuing Hardware Implementation," International Journal of Computer Science and Communication Security (IJCSCS), Vol.6, No.1, Jan., 2016.

[2] 3GPP's site: http://www.3gpp.org

[3] 3GPP TS 35.201 Version12.0.0, “3rd Generation Partnership Project; Technical Specification Group Services and System Aspects; 3G Security; Specification of the 3GPP Confidentiality and Integrity Algorithms; Document 1: 88 and 99 Specification," Sep., 2014.

[4] 3GPP TS 35.202 Version12.0.0, "3rd Generation Partnership Project; Technical Specification Group Services and System Aspects; 3G Security; Specification of the 3GPP Confidentiality and Integrity Algorithms; Document 2: KASUMI Specification,” Sep., 2014.

[5] Wikipedia, KASUMI's site: https://en.wikipedia.org/wiki/KASUMI

[6] Rabie A. Mahmoud, Magdy Saeb, "A Generalized Crypto Logic Unit (GCLU) with Software and Hardware Implementations," International Journal of Computer Science and Communication Security (IJCSCS), Vol.4, No.1, March, 2014.

[7] Magdy Saeb, "The Stone Cipher-192 (SC-192): A Metamorphic Cipher," International Journal of Computers and Network Security (IJCNS), Vol.1, No.2, pp.1-7, Nov., 2009.

[8] Rabie A. Mahmoud, Magdy Saeb, "Hardware Implementation of the Stone Metamorphic Cipher," International Journal of Computer Science and Network Security (IJCSNS), Vol.10, No.8, pp.54-60, 2010.

[9] Magdy Saeb, "Metamorphic Feistel Networks," International Journal of Computer Science and Communication Security (IJCSCS), Vol.5, No.3, July, 2015.

[10] Rabie A. Mahmoud, Magdy Saeb, “A Metamorphic-Enhanced Twofish Block Cipher And Associated FPGA Implementation," International Journal of Computer Science and Communication Security (IJCSCS), Vol.2, No.1, Jan., 2012.

[11] Ahmed Helmy, Magdy Saeb, A. Baith Mohamed, "A Metamorphic-Enhanced MARS Block Cipher," International Journal of Computer Science and
Communication Security (IJCSCS), Vol.3, No.4, July, 2013.

[12] Rabie A. Mahmoud, Magdy Saeb, "A Metamorphic-KeyHopping GOST Cipher and Its FPGA Implementation," International Journal of Computer Science and Communication Security (IJCSCS), Vol.3, No.7, Oct., 2013.

[13] Çetin Kaya Koç, “Cryptographic Engineering," Springer, 2009.

[14] Volnei A. Pedroni, "Circuit Design and Simulation with VHDL," 2nd Edition, MIT Press, 2010.

[15] Andrew Rushton, "VHDL for Logic Synthesis," 3rd Edition, John Wiley and Sons Ltd Publication, 2011.

[16] Altera's user-support site: https://www.altera.com/support/support-resources/designexamples/design-software/vhdl.html

[17] ModelSim-Altera's software-support site: https://www.altera.com/support/support-resources/designsoftware/modelsim.html

\section{Authors' Profiles}

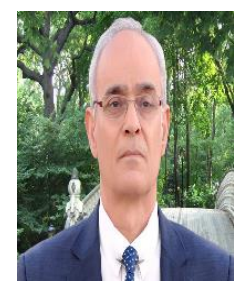

A. Baith MOHAMED received the BSc. in Computer Science, Vienna University, MSc. and Ph.D. in Computer Science Vienna University in 1992. He is a Professor at the Arab Academy for Science and Technology and Maritime Transport (AASTMT), Computer Engineering Department. In addition, he holds the position of Vice Dean for Training and Community Services, College of Engineering and Technology (2010). He is also get the position of Director of Arab Academy for Science and Technology and Maritime Transport, Latakia, Syria branch (2013). Now he is a President Councilor at the AASTMT in Alexandria Egypt. His research interests include computer and Network Security, Bioinformatics, Steganography, cryptography, and Genetic Algorithms. He was also a member of an International project team in Europe, for design and implementation and maintenance of subsystems in the environment of peripheral processor controls as part of a larger Public Switched Systems (EWSD) in SIEMENS, AG. Austria. Also, he was a scientific researcher in the department of Information Engineering, Seibersdorf Research Institute (Atomic Energy Agency) in Austria, for the design and implementation of security software system in the domain of railway automation project (VAX/VMS, DEC systems). He was also a member of software testing for distribution points in an international project in AEG, Vienna, Austria. He is a senior member of IEEE Computer Society, USA since 2001. baithmm@ @otmail.com

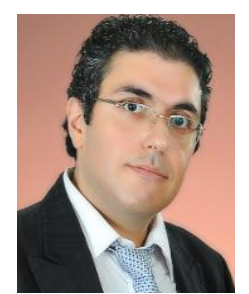

Rabie A. Mahmoud received the B.Sc. Degree, Faculty of Science, Tishreen University, Latakia-Syria, in 2001, the MS. and Ph.D. in Computational Science, Faculty of Science, Cairo University, Egypt, in 2007 and 2011 respectively. Currently, he is with General Organization of Remote Sensing (GORS), Syria and the Department of Computer Engineering, Arab Academy of Science, Technology \& Maritime Transport, Latakia, Syria branch. His current interests include Cryptography, FPGA Implementations of Cryptography and Data Security Techniques. rabiemah@yahoo.com 